\title{
X-RAY STANDING WAVES AND RUTHERFORD BACKSCATTERING STUDIES OF THE STRUCTURE OF Si SINGLE CRYSTALS IMPLANTED WITH Fe IONS
}

\author{
I.A. VARTANYANTZ \\ Institute of Crystallography RAS, Leninsky pr. 59, 117333 Moscow, Russia \\ J. Auleytner \\ Institute of Physics, Polish Academy of Sciences \\ Al. Lotników 32, 02-668 Warszawa, Poland \\ L. Nowicki, S. Kwiatkowski and A. Turos* \\ Soltan Institute for Nuclear Studies, Hoża 69, 00-681 Warszawa, Poland \\ and *Institute of Electronic Material Technology \\ Wólczyńska 133, 01-919 Warszawa, Poland
}

(Received February 5, 1996)

The X-ray standing wave and Rutherford backscattering spectroscopy in channelling geometry were applied for the investigation of the structure of silicon single crystals implanted with $80 \mathrm{keV}$ Fe ions. Both methods were used for the determination of crystal damage and lattice location of implanted metal atoms before and after thermal annealing. Both methods gave consistent results regarding the amorphization of $\mathrm{Si}$ due to the Fe-ion implantation. Moreover, using both methods some Fe substitution fraction was determined. The depth profiles of implanted atoms were compared to the results of computer simulations. Complementary use of $\mathrm{X}$-ray standing wave and Rutherford backscattering spectroscopy channelling techniques for studies of radiation damage and lattice location of implanted atoms is discussed.

PACS numbers: $61.10 .-\mathrm{i}$

\section{Introduction}

The practical applications of ion implantation in semiconductor technology have begun in the early 1970s. The knowledge of physical phenomena accompanying the implantation process is of great importance in designing and fabrication of silicon devices [1]. 
The X-ray standing wave (XSW) method considered for the first time for a perfect crystal by Batterman [2,3], is a powerful tool for investigation of the structure of real crystals [4] and the position of foreign (e.g. impurity) atoms in the subsurface layers of crystals. While measuring the fluorescence yield from impurity atoms by scanning in angle through a strong Bragg diffraction condition it is possible to localise, with a high accuracy, the atom position inside the crystal lattice. This method was used previously $[5,6]$ for structural investigations of $\mathrm{Si}$ crystals after being implanted with the different impurity atoms (As, Bi).

The XSW technique is nowadays widely used in a number of laboratories (see for e.g. reviews $[4,7])$. However, the interpretation of XSW results is in some cases quite a complicated and even not uniquely solvable problem [8]. For example in the case of implanted crystals not only the position of impurity atoms in the unit cell but also the structure (deformation and amorphization) of the crystal surface layer after the implantation process has to be taken into account. Therefore, for obtaining more reliable results it is quite helpful to combine the XSW method with some other complementary method. Rutherford backscattering spectroscopy in channelling geometry (RBS-c) is one of such methods that in combination with the XSW method can provide extensive information on the crystal surface layer structure as well as the lattice location of impurity atoms.

Preliminary results of XSW and RBS-c analysis of the silicon single crystals implanted with $\mathrm{Fe}$ and $\mathrm{Ni}$ ions were reported elsewhere $[9,10]$. The aim of this paper is to make a comprehensive analysis of the structure of iron implanted $\mathrm{Si}$ single crystals on the basis of these two methods.

The RBS-c method provides direct information about the depth distribution of the damage density of surface layers as well as the depth profile and lattice location of implanted atoms. This is due to the fact that the scattering yield of impurity atoms laying in atomic rows (along the studied channel) is strongly reduced and any impurity displacement can be detected by its increase [11]. In addition to these results, the XSW method can give additional information about the relaxation (deformation) of the surface layer as well as the information about the position of implanted atoms in the unit cell.

\section{Experimental}

Thick Si (111) oriented single crystals were prepared by mechanical polishing and etching. Next, the samples were implanted with Fe ions (dose $5 \times 10^{15} \mathrm{ions} / \mathrm{cm}^{2}$ at an energy of $80 \mathrm{keV}$ ). After ion implantation some samples were annealed at $750^{\circ} \mathrm{C}$ in the $\mathrm{N}_{2}$ atmosphere (details of the annealing process see in Ref. [9]).

The XSW experiment was carried out using the double-crystal non-dispersive scheme of symmetrical diffraction on the base of multicrystal spectrometer, designed and built in the Institute of Crystallography RAS (for references see e.g. $[4,12])$. The fluorescence radiation was registered by a $\mathrm{Si}(\mathrm{Li})$ solid state detector with the energy resolution of $200 \mathrm{eV}$. The $\mathrm{Cu} K_{\alpha}$ radiation (for excitation of $\mathrm{Fe} K_{\alpha}$ line) was used. The reflectivity and fluorescence yield curves were measured using the computer controlled multichannel analyser.

The RBS measurements were performed with $2 \mathrm{MeV}^{4} \mathrm{He}$ ions. The three-axis, computer controlled goniometer with $0.025^{\circ}$ angular resolution was used to per- 
form channelling measurements. To obtain sufficient depth resolution the geometry of normal incidence and glancing exit path was applied. The scattering angle was $105^{\circ}$.

\section{Results}

\section{1. $X S W$ results}

Figures 1a and $\mathrm{b}$ show the reflectivity curve and the $\mathrm{Fe} K_{\alpha}$ fluorescence yield curve from the crystal implanted with $\mathrm{Fe}$ ions before annealing as well as the results of theoretical calculations. The shape of the $\mathrm{Fe} K_{\alpha}$ fluorescence yield curve is quite similar to that of the reflectivity curve. One of the possible explanations of this experimental fact is that the implanted atoms are randomly distributed $[4,7]$.

Figure 2 shows the $\mathrm{Fe} K_{\alpha}$ fluorescence yield curve for the sample after annealing at $750^{\circ} \mathrm{C}$. The shape of the reflectivity curve does not change after annealing
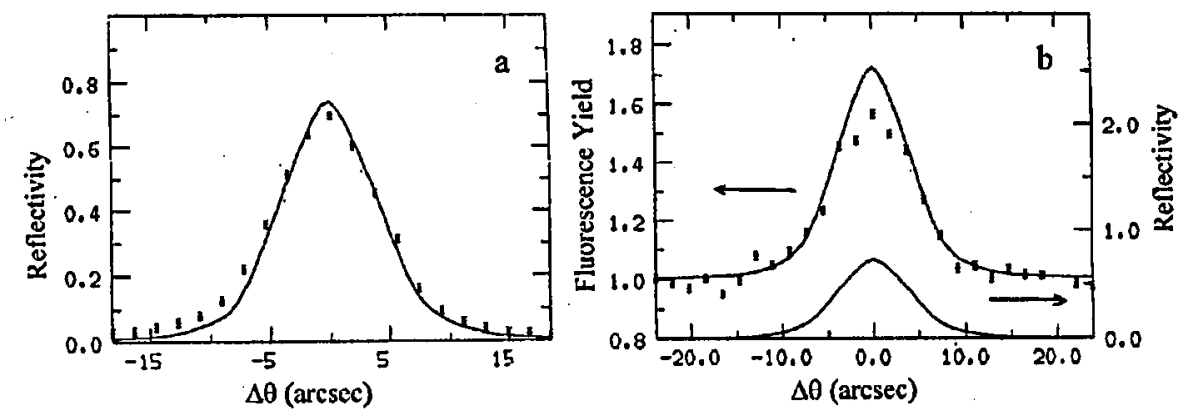

Fig. 1. (a) The reflectivity curve for $\mathrm{Si}(111)$ reflection, $\mathrm{Cu} K_{\alpha}$ radiation. Points experiment, solid line - theoretical curve. The theoretical curve was calculated for a perfect silicon crystal. (b) The fluorescence $\mathrm{Fe} K_{\alpha}$ yield curve for the as-implanted silicon sample. Solid lines - theory (parameters of the surface layers are listed in Table I).

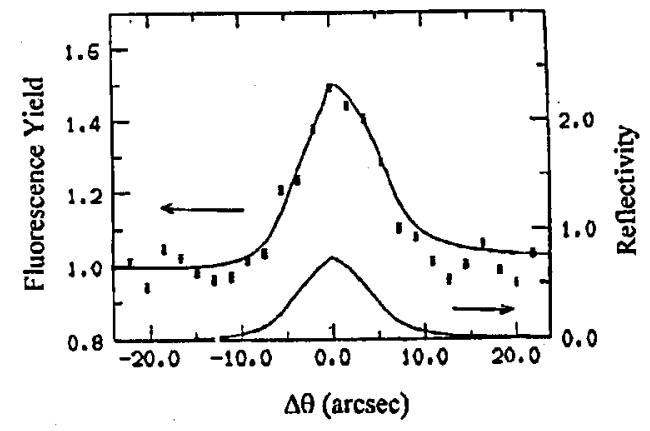

Fig. 2. The fluorescence $\mathrm{Fe} K_{\alpha}$ yield curve for the implanted silicon sample after annealing at $750^{\circ}$. Solid line - theory (parameters of the surface layers are listed in Table II). 
which indicates the stable quality of the host crystal. On the contrary, the shape of the fluorescence curve after annealing (Fig. 2) has been slightly changed comparing to that before annealing (Fig. 1b). This may indicate that after annealing a part of implanted atoms has moved to some regular e.g. substitutional, lattice sites and that the implanted part of crystal has undergone some relaxation. So, more sophisticated theoretical treatment is necessary in this case.

\section{2. $R B S-c$ results}

Figure 3a shows random and $\langle 111\rangle$ aligned backscattering spectra for the $\mathrm{Si}$ single crystal implanted with Fe ions to the fluence of $5 \times 10^{15} \mathrm{at} / \mathrm{cm}^{2}$. The large peak in the aligned spectrum extending from channel No. 330 to 380 has reached the random level thus indicating that the surface region was driven amorphously. The small peak appearing in the vicinity of channel 430 is due to the scattering by implanted Fe atoms. The properties of this peak will be discussed later on.

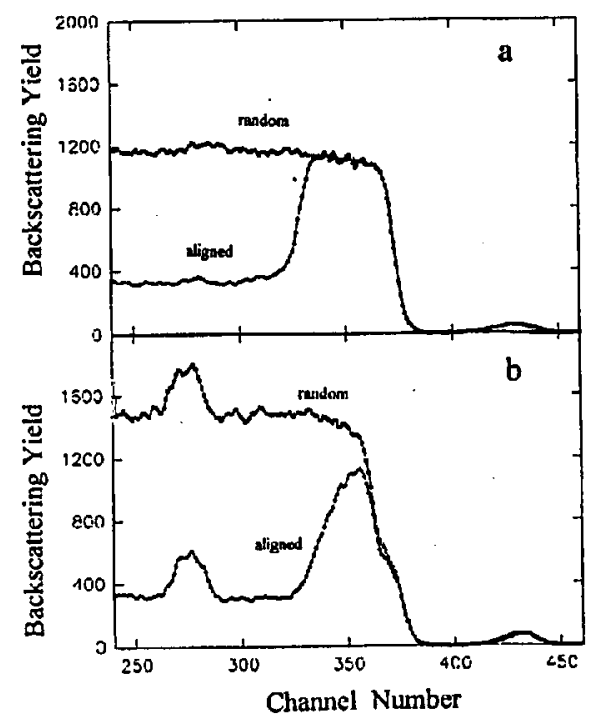

Fig. 3. Channelling and random spectra for $2 \mathrm{MeV}$ He ions scattered from silicon single crystals; (a) before annealing, (b) after annealing.

Important changes in the backscattering spectra were noticed after crystal annealing at $750^{\circ} \mathrm{C}$ as shown in Fig. 3b. First of all a peak appeared with the maximum located approximately at channel 275 . Since the position of this peak corresponds exactly to the channel, where the signal from oxygen atoms is expected, one can conclude that the crystal was partly oxidised during annealing. This was probably due to the low purity of the nitrogen gas used as the annealing atmosphere. The thickness of the oxide layer was estimated to be $40 \mathrm{~nm}$.

The damage peak in the aligned spectrum became narrower due to the shift of its low energy edge towards the higher channels. One can conclude that the crystal regrowth begins at the interface separating the crystalline and the amorphous 
regions and proceeds towards the crystal surface. In the region corresponding to the oxide layer, i.e. channels $360-380$, the aligned spectrum follows exactly the random one, thus indicating the absence of any crystalline structure in this region.

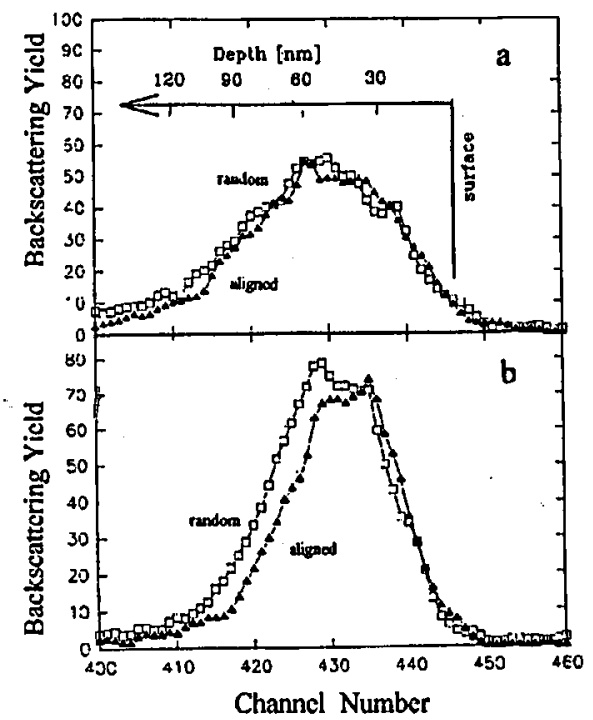

Fig. 4. Fe portions of channelling and random backscattering spectra; (a) before annealing, (b) after annealing.

The detailed structure of Fe-peaks before and after annealing is shown in Figs. 4a and b, respectively. For the as-implanted crystal Fe peaks for aligned and random orientation are identical which is the consequence of amorphization of implanted region. On the other hand, after annealing Fe atoms located in the deepest, already recovered region (channels 410-428) exhibit a substitutional fraction of about $30 \%$. One cannot expect any substitution of $\mathrm{Fe}$ atoms in the oxidised region and in fact the aligned and random spectra match well each other in the region of channels $428-447$.

\section{Analysis and discussion of results}

Figure 5a shows the results of the damage analysis from the spectra shown in Fig. 3, whereas Figs. 5b and $5 \mathrm{c}$ show the evolution of Fe atoms depth distributions. The standard measure of the damage density $k$ is given by the ratio of displaced atom density to the crystal atom density. Hence $k=100 \%$ denotes a complete amorphization. It was calculated according to the algorithm of Schmid [13]. Distributions in Fig. 3 were neither corrected for the detection system resolution nor for the energy straggling of analysing beam. The energy scale (channels) was converted into the depth scale. Damage distributions in Fig. 5a confirm quantitatively the direction of damage regrowth upon annealing as described in Sec. 3.

An important feature of the Fe-atom depth distribution for an as-implanted sample is its quasi-Gaussian shape with the maximum (i.e. Fe mean projected 


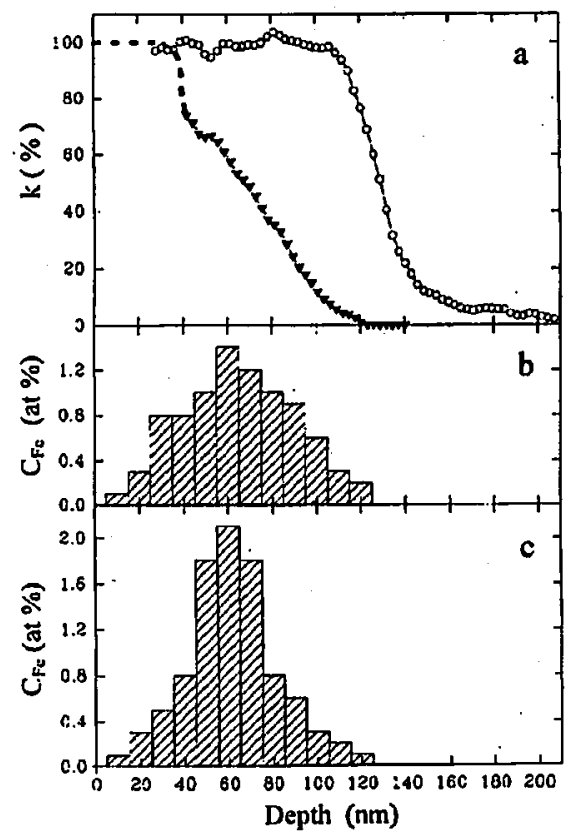

Fig. 5. (a) Damage density in silicon due to $80 \mathrm{keV}$ Fe ion implantation for the as-implanted sample (circles) and for the annealed sample (triangles). (b) Profile of Fe impurity concentration for the as-implanted sample. (c) Profile of Fe impurity concentration for the annealed sample.

range) located at the depth of $70 \mathrm{~nm}$ and range straggling of $80 \mathrm{~nm}$. One notes that the amorphized region extends over $190 \mathrm{~nm}$. Upon annealing the Fe distribution became narrower, however, its maximum position did not change. Also the peak area has remained the same. This effect is apparently due to the low solubility of $\mathrm{Fe}$ in silicon. In such a case the regrowth front pushes away impurity atoms in concentrations exceeding the solubility limit. In such a case the impurity segregation occurs in a not yet regrown region. This leads to the enhanced concentration of $\mathrm{Fe}$ atoms in the near surface region. It should be pointed out that the oxidised region forms a diffusion barrier for the further diffusion of Fe atoms.

The XSW experimental results were analysed on the basis of the general theory of X-ray standing waves in deformed crystals [14, 4]. For theoretical fitting of the angular dependences of the fluorescence yield for implanted region of the silicon sample a "layered" model was used (see for details $[9,15,16])$. The same model was applied for the calculation of the reflectivity curve but it has not changed the shape of the curve. The main reason for this is due to the fact that the thickness of the implanted layer $L$ (in our case $L \cong 0.1 \div 0.2 \mu \mathrm{m}$ ) is much smaller than the extinction length $L_{\text {ex }}\left(L_{\mathrm{ex}}=1.51 \mu \mathrm{m}\right)$. In the frame of this model each deformed layer is characterised by its constant value of thickness $d$, deformation $\Delta d / d$ and amorphization factor $\exp (-W)$. Foreign atoms in these layers are deter- 
mined by two main parameters: $Z_{\mathrm{c}}^{\mathrm{Fe}}-$ the coherent position, i.e. the position of the mean plane of the impurity atoms with respect to the diffraction planes of the crystal and $F_{c}^{\mathrm{Fe}}$ - the coherent fraction, which describes the static and thermal displacement of the atoms from the mean position $Z_{\mathrm{c}}^{\mathrm{Fe}}$. Naturally the contribution of each layer to the secondary radiation (fluorescence) yield is determined by the distribution (concentration $n_{c}^{\mathrm{Fe}}$ ) of the impurity atoms in the surface layer of the silicon sample.

\section{TABLE I}

Parameters of the layers for theoretical fitting of

Fe implanted silicon samples before annealing.

\begin{tabular}{c|l|c|c|c}
\hline \hline$N^{\circ}$ & $d[\mu \mathrm{m}]$ & $\exp (-W)$ & $F_{\mathrm{c}}^{\mathrm{Fe}}$ & $n_{\mathrm{c}}^{\mathrm{Fe}}[\%]$ \\
\hline 1. & 0.015 & 0 & $0.0 \pm 0.02$ & 5 \\
2. & 0.01 & 0 & $0.0 \pm 0.02$ & 20 \\
3. & 0.02 & 0 & $0.0 \pm 0.02$ & 60 \\
4. & 0.01 & 0 & $0.0 \pm 0.02$ & 70 \\
5. & 0.01 & 0 & $0.0 \pm 0.02$ & 100 \\
6. & 0.01 & 0 & $0.0 \pm 0.02$ & 85 \\
7. & 0.02 & 0 & $0.0 \pm 0.02$ & 70 \\
8. & 0.01 & 0 & $0.0 \pm 0.02$ & 40 \\
9. & 0.01 & 0.05 & $0.0 \pm 0.02$ & 20 \\
10. & 0.01 & 0.20 & $0.0 \pm 0.02$ & 15 \\
11. & 0.01 & 0.54 & - & - \\
12. & 0.01 & 0.80 & - & - \\
13. & 0.02 & 0.90 & - & - \\
14. & 0.045 & 0.95 & - & -
\end{tabular}

For the theoretical fitting of the $\mathrm{Fe} K_{\alpha}$ fluorescence yield curve from the crystal implanted with Fe ions before annealing a 14-layer model of the implanted surface of silicon was used. The parameters of the layers are listed in Table I. The profile of amorphization $(\exp (-W))$ of the silicon surface layer and the distribution of implanted $\mathrm{Fe}$ atoms $n_{\mathrm{c}}^{\mathrm{Fe}}$ were taken from the results of the RBS-c experiment (Fig. 5). The best fitting for experimental results (solid curve in Fig. 1b) was obtained with the value of the coherent fraction $F_{\mathrm{c}}^{\mathrm{Fe}}=0.00 \pm 0.02$ (there is a small discrepancy between theoretical curve and central experimental points that may be due to some experimental problems). This value, as it was expected, means that the implanted atoms occupy random positions in the lattice cell. Due to total amorphization of the surface layer and random position of impurity atoms we cannot speak about any deformation in this surface layer or mean position of the Fe atoms (that is why they are not tabulated in Table I). 
TABLE II

Parameters of the layers for theoretical fitting of Fe implanted silicon samples before annealing.

\begin{tabular}{c|c|c|c|c|c|c}
\hline \hline$N^{\circ}$ & $d[\mu \mathrm{m}]$ & $\Delta d / d\left(\times 10^{4}\right)$ & $\exp (-W)$ & $F_{\mathrm{c}}^{\mathrm{Fe}}$ & $Z_{\mathrm{c}}^{\mathrm{Fe}}$ & $n_{\mathrm{c}}^{\mathrm{Fe}}[\%]$ \\
\hline 1. & 0.015 & 0 & 0 & 0 & 0 & 4 \\
2. & 0.01 & 0 & 0 & 0 & 0 & 14 \\
3. & 0.01 & 0 & 0 & 0 & 0 & 24 \\
4. & 0.01 & $-9.6 \pm 0.09$ & 0.15 & $0.27 \pm 0.02$ & 0 & 38 \\
5. & 0.01 & $-9.6 \pm 0.09$ & 0.34 & $0.27 \pm 0.02$ & 0 & 86 \\
6. & 0.01 & $-9.6 \pm 0.09$ & 0.41 & $0.27 \pm 0.02$ & 0 & 100 \\
7. & 0.01 & $-9.6 \pm 0.09$ & 0.54 & $0.27 \pm 0.02$ & 0 & 86 \\
8. & 0.01 & $-9.6 \pm 0.09$ & 0.64 & $0.27 \pm 0.02$ & 0 & 40 \\
9. & 0.01 & $-9.6 \pm 0.09$ & 0.77 & $0.27 \pm 0.02$ & 0 & 30 \\
10. & 0.01 & $-9.6 \pm 0.09$ & 0.88 & $0.27 \pm 0.02$ & 0 & 14 \\
11. & 0.02 & $-9.6 \pm 0.09$ & 0.97 & $0.27 \pm 0.02$ & 0 & 7
\end{tabular}

For the theoretical fitting of the $\mathrm{Fe} K_{\alpha}$ fluorescence yield curve for the same crystals after annealing an 11-layer model of the implanted surface of silicon was used. The parameters of the layers are presented in Table II. As in the previous case the profile of amorphization of the silicon surface and the distribution of implanted atoms $\left(n_{\mathrm{c}}^{\mathrm{Fe}}\right)$ were taken from the results of the RBS-c experiment (Fig. 5). In addition it was proposed that some parts of implanted atoms after annealing locate in substitution positions of $\mathrm{Si}$ atoms (parameter $Z_{\mathrm{c}}^{\mathrm{Fe}}=0$ ). Due to the experimental result that the surface layer $\cong 40 \mathrm{~nm}$ is oxidised we have taken the amorphization factor $\exp (-W)$ equal to zero on the top of the annealed sample (see Table II). During the fitting procedure the coherent fraction of Fe atoms in the corresponding layers and the value of deformation of the surface layer were varied. To minimise the number of fitting parameters we have taken the uniform distribution of $F_{c}^{\mathrm{Fe}}$ and $\Delta d / d$ in the surface layer for the depths $\cong 40 \div 120 \mathrm{~nm}$ (we have taken the simplest model because of the small change of the fluorescence yield curve after annealing (Fig. 2)). The best fitting results were obtained for the parameters listed in Table II (solid curve in Fig. 2). As it follows from these results the average value of the surface relaxation is equal to $\Delta d / d=(-9.6 \pm 0.09) \times 10^{-4}$ and the value of the coherent fraction is equal to $F_{c}^{\mathrm{Fe}}=0.27 \pm 0.02$. This value of the coherent fraction means that approximately $27 \%$ (with respect to diffraction planes) of implanted Fe atoms are in substitution Si positions. It is interesting to point out here that from the independent XSW analysis we have obtained practically the same value of substitutional fraction of $\mathrm{Fe}$ atoms as after the RBS-c analysis (see the end of Sec. 3).

The obtained results show the effectiveness and mutual complement of the two methods for investigation of implanted crystals. The RBS-c experiments directly give the depth profiles of damage density for Si surface layer and the profile 
of implanted ions concentration. The XSW analysis is the precise phase sensitive method. It gives directly the value of the phase $\phi(z)$ that is in our case equal to $h \cdot u(z)+P_{\mathrm{c}}$, where $u(z)$ is the deformation of the surface layer (in the case of the uniform deformation we have $u_{z}=(\Delta d / d L)$ and $P_{c}=h_{z} Z_{c}$. As we could see from this expression the phase is the sum of deformation and the position of the impurity atoms and we need some additional information to determine these two values independently. As it was shown in this paper the combination of RBS-c and XSW analysis gives us the opportunity to solve this problem and determine the position of impurity atoms in the unit cell as well as the total deformation (relaxation) of the surface layer.

\section{Acknowledgments}

The authors are thankful to Prof. M.V. Kovalchuk for his permanent interest and assistance during the performance of this work. The authors are thankful to Dr. D. Żymierska for her assistance and help during the preparation of the manuscript.

\section{References}

[1] A. Davies, J.M. Mayer, J.H. Johansen, Ion Implantation in Semiconductors, McGraw Hill, New York 1971.

[2] B.W. Batterman, Phys. Rev. 133, A759 (1964).

[3] B.W. Batterman, H. Cole, Rev. Mod. Phys. 36, 681 (1964).

[4] M.V. Kovalchuk, V.G. Kohn, Usp. Fiz. Nauk 149, 69 (1986) [Sov. Phys.-Usp. 29, $426(1986)]$.

[5] J.A. Golovchenko, B.W. Batterman, W.L. Brown, Phys. Rev. B 10, 4239 (1974).

[6] N. Hertel, G. Materlik, J. Zegenhagen, Z. Phys. B, Condens. Matter 58, 199 (1985).

[7] J. Zegenhagen, Surface Science Reports 18, 271 (1993).

[8] I.A. Vartanyantz, M.V. Kovalchuk, V.G. Kohn, A.M. Nikolaenko, I.Yu. Kharitonov, Pis'ma Zh. Eksp. Teor. Fiz. 40, 630 (1989) [Sov. Phys. JETF Lett. 49, 726 (1989)].

[9] I.A. Vartanyantz, M.V. Kovalchuk, A.N. Sosphenov, J. Auleytner, J. Majewski, Acta Phys. Pol. A 80, 811 (1991).

[10] L. Nowicki, S. Kwiatkowski, A. Turos, J. Auleytner, I.A. Vartanyantz, M.V. Kovalchuk, A.N. Sosphenov, Nucleonica 39, No 3, 49 (1994).

[11] C. Feldman, J.W. Mayer, Materials Analysis by Ion Channeling, Academic Press, New York 1984.

[12] Z.G. Pinsker, Rentgenovskaya kristallooptika (X-Ray Crystal Optics), Nauka, Moscva 1982 (in Russian).

[13] K. Schmid, Radiation Effects 17, 201 (1973).

[14] A.M. Afanas'ev, V.G. Kohn, Zh. Eksp. Teor. Fiz. 74, 300 (1978) [Sov. Phys..JETP 47, $154(1978)]$.

[15] V.G. Kolın, M.V. Kovalchuk, Phys. Status Solidi A 64, 369 (1981).

[16] M.V. Kovalchuk, V.G. Kohn, E.F. Lobanovich, Fiz. Tverd. Tela 27, 3379 (1985) [Sov. Phys. Solid State 27, 2875 (1985)]. 